\title{
Renormalized jellium model for charge-stabilized colloidal suspensions
}

\author{
Emmanuel Trizac ${ }^{1}$ and Yan Levin ${ }^{2,1}$ \\ ${ }^{1}$ Laboratoire de Physique Théorique, UMR CNRS 8627, Université de Paris XI, Bâtiment 210, F-91405 Orsay Cedex, France \\ ${ }^{2}$ Instituto de Física, Universidade Federal do Rio Grande do Sul, Caixa Postal 15051, CEP 91501-970 Porto Alegre, RS, Brazil
}

(Received 30 July 2003; published 19 March 2004)

\begin{abstract}
We introduce a renormalized jellium model to calculate the equation of state for charged colloidal suspensions. An almost perfect agreement with Monte Carlo simulations is found. Our self-consistent approach naturally allows to define the effective charge of particles at finite colloidal density. Although this quantity may differ significantly from its counterpart obtained from the standard Poisson-Boltzmann cell approach, the osmotic pressures for both models are in good agreement. We argue that by construction, the effective charge obtained using the jellium approximation is more appropriate to the study of colloidal interactions. We also discuss a possibility of a fluid-fluid critical point and show how our equation of state can be used to shed light on the surprising results found in recent sedimentation experiments.
\end{abstract}

DOI: 10.1103/PhysRevE.69.031403

PACS number(s): 82.70.-y, 61.20.Gy, 64.70.-p

In spite of the great effort invested in trying to understand the phase stability of colloidal suspensions, our knowledge of these complex systems is still quite rudimentary. It is curious to compare this situation with an earlier debate concerning the nature, or even the possibility of the liquid-gas phase separation in symmetric electrolytes. Now this debate is almost over, and the phase structure of a symmetric 1:1 electrolyte is well elucidated, although the universality class of the critical point is still being discussed. The Coulombic criticality-to distinguish it from the solvophobic criticality - cannot be observed in water and organic solvents of low dielectric permittivity must be used [1]. The phase separation results from the strong electrostatic correlations between the cations and anions of electrolyte [2].

The phase stability of charged colloidal suspensions, on the other hand, is far from being well understood [3-10]. The strong charge and size asymmetry between the macroions and the microions present in a suspension makes it very difficult to apply to these systems the traditional tools of the liquid state theory. Nevertheless, in view of results on symmetric 1:1 electrolytes, it would not be very surprising if colloidal suspensions inside a solvent of sufficiently low dielectric permittivity $\epsilon$ also presented a gas-liquid phase transition. Indeed such an instability has been observed in recent Monte Carlo simulations, see for example Refs. [4,11] and references therein. What is much more surprising is that there are some experimental indications of an instability even in aqueous suspensions containing only monovalent counterions. Theoretical estimates of the strength of electrostatic correlations for aqueous suspensions suggest that they should be too "hot" for an instability to set in. Nevertheless the experimental situation remains unclear [12].

A number of theories have been proposed to address this unsettling experimental situation. A major drawback of most of these approaches is that they rely on uncontrolled approximations which have not been fully tested. However, numerical solution of the full nonlinear Poisson-Boltzmann (PB) equation inside a spherical Wigner-Seitz (WS) cell [13] finds no indication of any thermodynamic instability [7]. Of course, one can rightly question the reliability of the WignerSeitz cell PB model for the study of a fluid phase of a highly disordered suspension. On the one hand, since the main contribution to the osmotic pressure inside an aqueous suspension comes from the polyion-microions interactions, the cell might not be such a bad approximation. On the other hand, the cell fails to properly account for the colloid-colloid correlation, but these might not be of much importance for aqueous suspensions with monovalent counterions. Nevertheless, while the cell model is a good approximation for dense colloids, one should be very careful in extrapolating its findings to highly dilute suspensions. Clearly there is an urgent need for an accurate theory which will not rely on the cell approximation and which would be relevant for the study of colloidal phase stability. In this paper we shall present such a theory. Our approach is similar to the jellium approximation much used in the solid state physics.

Consider an aqueous suspension of colloidal particles of charge $-Z q$ and radius $a$ in contact with a reservoir of monovalent salt at concentration $c_{s}$ and electrostatic potential $\phi_{r}=0$ ( $q$ is the elementary charge). The number of counterions and coions inside the suspension is determined by the thermodynamic equilibrium. While the colloidal particles are more or less uniformly distributed throughout the solution-we are mainly interested in the small density regime - the positions of counterions and coions are strongly correlated with the positions of polyions. As a leading order approximation we can, therefore, take the polyion-polyion correlation function to be $g_{p p}=1$ [14] while the exact polyion-counterion and polyion-coion correlation functions are $g_{p \pm}=e^{-\beta w_{ \pm}(r)}$, where $w_{ \pm}(r)$ are the polyion-counterion and the polyion-coion potentials of mean force and $\beta$ $=1 /\left(k_{B} T\right)$ is the inverse temperature.

Choosing the coordinate system in such a way that it is centered on top of one of the colloidal particles, the electrostatic potential satisfies the Poisson equation

$$
\nabla^{2} \phi=-\frac{4 \pi}{\epsilon} \rho_{q}(r)
$$

The charge density is $\rho_{q}(r)=-\rho_{\text {back }}+q \rho_{+}(r)-q \rho_{-}(r)$, where $\rho_{\text {back }}=Z_{\text {back }} q \rho_{p}$ and $\rho_{p}$ is the mean density of colloids inside suspension. The background charge is excluded from 
the colloidal interior. Naively one can suppose that $Z_{\text {back }}$ $=Z$. This, however, is not correct and the bare charge must be renormalized in such a way as to lead to a self-consistent solution of Eq. (1), as discussed below.

Approximating the potential of mean force by the electrostatic potential, the local concentration of counterions and coions inside a suspension is

$$
\rho_{ \pm}(r)=c_{s} e^{\mp \beta q \phi(r)} .
$$

It is important to keep in mind that in order for suspension to be neutral, the electrostatic potential at infinity (bulk) cannot vanish but must saturate to a value $\phi(\infty)=\phi_{D}$ given by $c_{s} \sinh \left[\beta q \phi_{D}\right]=\rho_{p} Z_{\text {back }}$. There exists, therefore, an electrostatic potential difference between the suspension and the salt reservoir. In the biophysics literature, this potential difference is known as the Donnan potential, and is partially responsible for the biological cell transmembrane potential.

Far away from the colloidal surface, the electrostatic potential reduces to the familiar Debye-Hückel expression

$$
\phi(r)=\phi_{D}-\frac{Z_{\text {eff }} q}{\epsilon(1+\kappa a) r} e^{-\kappa(r-a)},
$$

where $\kappa^{2}=4 \pi \lambda_{B}\left[\rho_{+}(\infty)+\rho_{-}(\infty)\right]$ and $\lambda_{B}=\beta q^{2} / \epsilon$ denotes the Bjerrum length. The value of $Z_{\text {eff }}$ is determined selfconsistently from the numerical solution of Eq. (1) so that $Z_{\text {eff }}\left(Z, Z_{\text {back }}, c_{s}\right)=Z_{\text {back }}$. This renormalization of background charge is a consequence of counterion condensation. It is important to keep in mind that for highly charged colloidal particles, $Z_{\text {eff }}$ is not equal to the bare colloidal charge. Furthermore, using the contact theorem and the vanishing of the electric field as $r \rightarrow \infty$, the osmotic pressure within the suspension takes a simple form

$$
\beta P=\rho_{p}+\rho_{+}(\infty)+\rho_{-}(\infty)-2 c_{s}=\rho_{p}+\sqrt{Z_{\mathrm{eff}}^{2} \rho_{p}^{2}+4 c_{s}^{2}}-2 c_{s} .
$$

It is a nice feature of the jellium approximation that once the effective charge is determined, the Debye length $1 / \kappa$ and the osmotic pressure both follow directly.

In Fig. 1 we compare the osmotic pressures calculated using the renormalized jellium approximation to the ones obtained within the WS cell (for both approaches, the effective charges and pressures become independent of $Z$ when the latter is large enough, approximately $Z>20 a / \lambda_{B}$; Fig. 1 has been obtained under this condition of saturation, which is usually met in colloidal suspensions). A surprisingly good agreement is found between the two theories, with a discrepancy only for volume fractions $\eta>0.15$. In Fig. 2, the osmotic pressure calculated in the renormalized jellium model is compared to the results of recent Monte Carlo simulations [4] (where a model system of charged spherical macroions and point counterions interacting solely through hard sphere and Coulomb forces has been considered). The agreement is excellent, and justifies the neglect of colloid-colloid correlations in our treatment. Clearly, for high packing fractions (namely $\eta>0.1$ ) colloid-colloid correlations become important and should invalidate our approach.

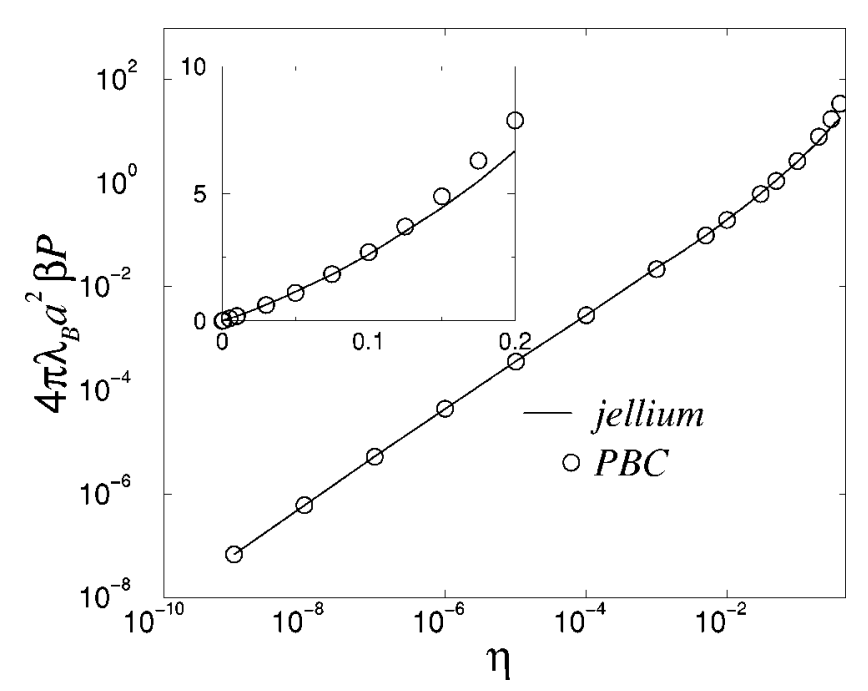

FIG. 1. Pressure-volume fraction isotherms within the PoissonBoltzmann cell (PBC) model and the renormalized jellium approximation for $c_{s}=0$ (deionized situation). The packing fraction is defined as $\eta=4 \pi \rho_{p} a^{3} / 3$. The inset shows the same data on the linear scale.

In Fig. 3, the effective charges calculated using the cell model [15] and the renormalized jellium theory are compared. At very low volume fractions $\left(\eta<10^{-5}\right)$ the saturated effective charge of a salt-free suspension is in perfect agreement with the value of saturated effective charge found within the WS cell and can be approximated by a simple equation,

$$
Z_{\mathrm{sat}} \approx \frac{a}{\lambda_{B}}[\delta-\gamma \ln (\eta)],
$$

where $\gamma \approx 1$ and $\delta \approx 2$. For volume fractions $\eta>10^{-4}$, there is a fairly strong disagreement between the effective charges

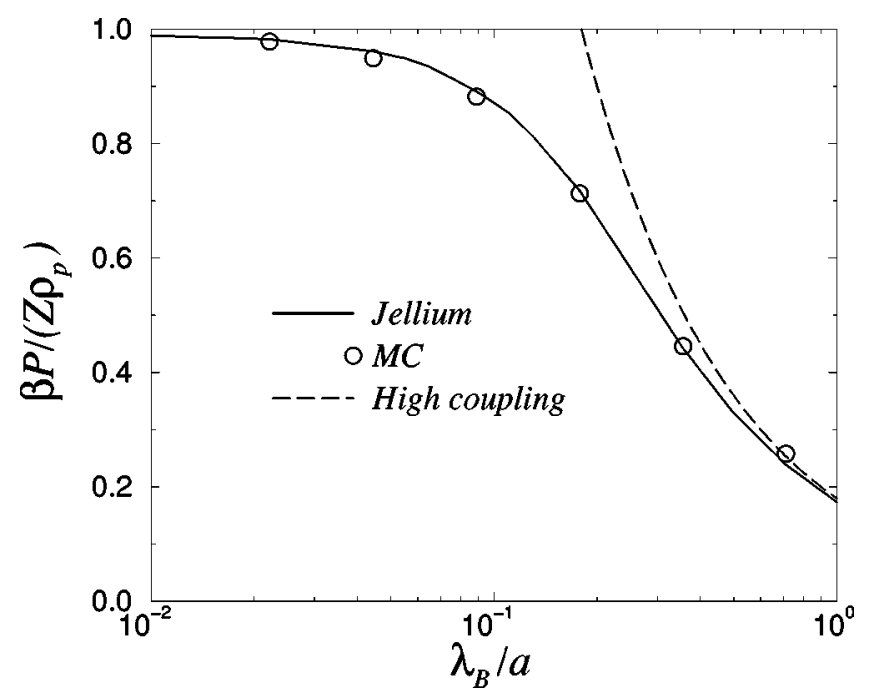

FIG. 2. Comparison between the osmotic coefficient $\beta P /\left(Z \rho_{p}\right)=Z_{\text {eff }} / Z$ calculated using Monte Carlo (MC) simulations [4] and the renormalized jellium model for $c_{s}=0, Z=40, \eta$ $=0.00125$. At large couplings, we predict $\beta P /\left(Z \rho_{p}\right)$ $\approx 0.1802 a / \lambda_{B}$, shown as the dashed curve. 


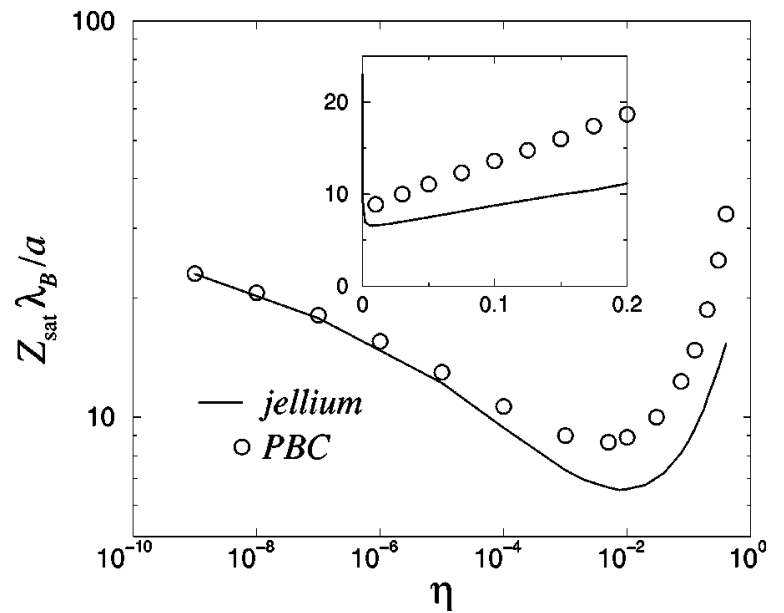

FIG. 3. Effective values of colloidal charges calculated with the Poisson-Boltzmann cell model [15] and the renormalized jellium approximation for $c_{s}=0$. Inset shows the same data but on a linear scale.

predicted by the two theories, even if both models are in very good agreement for the value of the osmotic pressure.

If the effective charge is to be used to study the structural properties of colloidal suspensions (such as the structure factors or any other quantity requiring the knowledge of the effective interaction potential between two polyions), we argue that the jellium effective charge is the more relevant quantity. The reason for this is that within the jellium approximation, two colloids at large separations $R$ interact by the usual Derjaguin-Landau-Verwey-Overbeek (DLVO) potential, as follows after some algebra from integrating the stress tensor over a colloid's surface (see also Ref. [2]),

$$
V(R)=\frac{Z_{\mathrm{eff}}^{2} q^{2}}{\epsilon(1+\kappa a)^{2} R} e^{\kappa(2 a-R)}
$$

This is not the case for two colloids inside a WS cell for which the interaction potential is a very complicated function of separation and can only be calculated numerically. In fact, for separations larger than the interparticle distance, it is even difficult to properly define what one means by an interaction potential between colloids within the WS formalism, since the overall charge neutrality results in a vanishing electrostatic interaction between the two cells.

To assess the quantitative validity of our approach, we compare our (saturated) effective charges to those deduced in recent experiments using a torsional resonance spectroscopy [16]. For colloidal volume fractions in the interval $10^{-3}$ $<\eta<3.10^{-2}, Z_{\text {sat }} \lambda_{B} / a$ was found to be close to 6 [16]. This is in much better agreement with the renormalized jellium model, which finds for these volume fractions $Z_{\mathrm{sat}} \lambda_{B} / a$ varying between 6.7 and 7.0, as compared to the WS cell prediction of 8.7-9. We also note that a value close to 6 has been reported for this density regime in the Monte Carlo study presented in Ref. [17].

We also emphasize that within the jellium model, the inverse screening length $\kappa$ is naturally related to the effective salt density [see the definition below Eq. (3)]. This should be contrasted with the cell model, for which there is no simple connection between the two quantities.

The jellium model predicts stability of a charged colloidal suspension against a fluid-fluid phase separation. This reinforces the cell picture, where no instability is found. Our approach, however, neglects microionic correlations, which become important at high electrostatic couplings $[2,18,19]$, more precisely when $\Gamma=v^{2} \lambda_{B} / d$ exceeds a threshold close to $2, d$ being a characteristic distance between microions in the electric double layer and $v$ their valency. In practice, however, ionic hydration puts a lower bound to $d$, which prevents the high coupling regime from ever being reached in water with monovalent counterions. Under these conditions our approach should, therefore, be quite reliable. Alternatively, when dealing with point particles, $d$ may be estimated as $d \simeq\left(4 \pi a^{2} v / Z\right)^{1 / 2}[2,18]$. For the Monte Carlo simulations reported in Fig. 2, $\Gamma=2$ therefore corresponds to an instability threshold $\lambda_{B} / a \simeq 1.1$. Beyond this point, the microionic correlations destabilize the system and lead to a fluid-fluid separation [4,11]. Nevertheless, the pressures obtained within the renormalized jellium approximation are in very good agreement with the Monte Carlo simulations, even at fairly large electrostatic couplings (in Fig. 2, we have included the highest coupling for which the pressure was computed in Ref. [4]). We therefore expect that the jellium approximation, suitably corrected to include the counterion induced attraction between the colloidal particles present at strong couplings, might be sufficient to account for the thermodynamic instability in suspensions containing multivalent ions. Work along these lines is in progress.

Finally, it is interesting to speculate how the renormalized jellium model can help to shed light on the problem of sedimenting colloidal dispersions [20-24]. Recently, Philipse and Koenderink (PK) [25] observed strongly inflated concentration profiles for charged monodispersed colloidal particles in absolute ethanol. Use of ethanol, instead of water, allows to produce highly deionized suspensions with "salt" concentrations on the order of $10^{-9} M$. The renormalized jellium predicts that for this salt concentration, an infinitely dilute colloid would have an effective charge of $Z_{\text {eff }} \approx 20 a / \lambda_{B}$. It is difficult to know what is precisely the bare charge of colloidal particles inside ethanol; however, since the Bjerrum length in ethanol is $\lambda_{B} \approx 2.3 \mathrm{~nm}$, a fairly small bare charge on the order of a few hundred electrons is enough to place the particles (with radius $a \simeq 90 \mathrm{~nm}$ [25]) in the saturation regime. Using this observation we can partially account for the observations of PK.

Static equilibrium of a suspension in a gravitational field requires that

$$
\frac{d P}{d x}=-m g \rho_{p},
$$

where $m$ is the colloidal mass (corrected for buoyancy), $g$ the gravitational acceleration, and $x$ the vertical displacement. It is convenient to define the gravitational length as $l_{g}$ $=1 /(m g \beta)$, which for the experiments of $\mathrm{PK}$ is $l_{g}$ $\approx 0.2 \mathrm{~mm}$ [25]. Substituting the expression for the osmotic pressure Eq. (4) into Eq. (7) and using the dependence of the 
saturated effective charge on the colloidal volume fraction, Eq. (7) can be integrated. We find that for colloidal volume fractions $\eta>\eta_{1}=10^{-5}$ (i.e., in the counterion dominated regime)

$$
\ln \left(\eta / \eta_{0}\right)-\frac{1}{2}\left[\ln ^{2}(\eta)-\ln ^{2}\left(\eta_{0}\right)\right]=\frac{\lambda_{B} x}{a l_{g}},
$$

where $\eta_{0}$ is the reference volume fraction at the $x$ origin. This can be taken as $\eta_{0} \approx 0.01$, the point at which the hardcore effects are completely negligible, and one can be sure to be looking at the dilute tail of the sedimenting profile. Note that for small volume fractions, $\eta \sim \exp \left(-\sqrt{2 \lambda_{B} x / a l_{g}}\right)$ unlike the simple exponential barometric law. For $\eta<\eta_{\times} \approx 5$ $\times 10^{-8}$, the salt resulting from solvent dissociation dominates over counterions, and we recover the usual barometric law $\eta(x) \sim \exp \left(-x / l_{g}\right)$. Results concerning the crossover regime $\eta_{\times}<\eta<\eta_{1}$ will be published elsewhere. It is interesting to estimate the extent of the sedimentation profile predicted by the renormalized jellium model. Since the concentrations of colloids on the order of $\eta_{m} \approx 10^{-5}$ are still detectable, we find that the profile extends distance $x$ $\sim a l_{g} \ln ^{2}\left(\eta_{m}\right) /\left(2 \lambda_{B}\right) \approx 2600 l_{g}$. For the colloids used by PK, this is almost $50 \mathrm{~cm}$. Hence, the observed inflation of the sedimentation profile.
To conclude, we have proposed a self-consistent renormalized nonlinear jellium model that constitutes an alternative to the widely used PB cell approach for charged colloidal suspensions. Surprisingly, the equations of state within the two theories turn out to be very close, even though the approximations involved to account for finite colloidal density are very different (finite cell against a renormalized background). Our results point to the relevance of the cell picture even at extremely low densities. This reinforces the argument that a fluid-fluid phase instability is impossible, for highly charged colloids in water at room temperature, as long as the microions are monovalent [which allows to neglect microionic correlations, thereby identifying the potential of mean force with the electrostatic potential, see Eq. (2)]. Our approach also allows to define in a natural way, not only the effective charge of the macroions, but also the effective screening length; these quantities are experimentally and conceptually more relevant than those obtained within the cell approach. Finally, our equation of state for deionized systems helps in understanding recent sedimentation experiments where "anomalous" density profiles have been reported.

This work was supported in part by the Brazilian agencies CNPq and FAPERGS and by the french CNRS.
[1] M.E. Fisher, J. Stat. Phys. 75, 1 (1994).

[2] Y. Levin, Rep. Prog. Phys. 65, 1577 (2002).

[3] R. van Roij, M. Dijkstra, and J.-P. Hansen, Phys. Rev. E 59, 2010 (1999).

[4] P. Linse, J. Chem. Phys. 113, 4359 (2000).

[5] P. Warren, J. Chem. Phys. 112, 4683 (2000).

[6] A. Diehl, M.C. Barbosa, and Y. Levin, Europhys. Lett. 53, 86 (2001).

[7] M. Deserno and H.H. von Grünberg, Phys. Rev. E 66, 011401 (2002).

[8] G. Téllez and E. Trizac, J. Chem. Phys. 118, 3362 (2003).

[9] M.N. Tamashiro and H. Schiessel, J. Chem. Phys. 119, 1855 (2003).

[10] J.-F. Dufrêche, T.O. White, and J.-P. Hansen, Mol. Phys. 101, 1741 (2003).

[11] P. Linse and V. Lobaskin, Phys. Rev. Lett. 83, 4208 (1999).

[12] A. van Blaaderen (unpublished).

[13] R.A. Marcus, J. Chem. Phys. 23, 1057 (1955).

[14] B. Beresford-Smith, D.Y.C. Chan, and D.J. Mitchell, J. Colloid
Interface Sci. 105, 216 (1985).

[15] S. Alexander, P.M. Chaikin, P. Grant, G.J. Morales, P. Pincus, and D. Hone, J. Chem. Phys. 80, 5776 (1984).

[16] P. Wette, H.J. Shöpe, and T. Palberg, J. Chem. Phys. 116, 10 981 (2002).

[17] M.J. Stevens, M.L. Falk, and M.O. Robbins, J. Chem. Phys. 104, 5209 (1996).

[18] I. Rouzina and V.A. Bloomfield, J. Phys. Chem. 100, 9977 (1996).

[19] B.I. Shklovskii, Phys. Rev. Lett. 82, 3268 (1999).

[20] R. Piazza, T. Bellini, and V. Degiorgio, Phys. Rev. Lett. 71, 4267 (1993).

[21] T. Biben and J.P. Hansen, J. Phys.: Condens. Matter 6, A345 (1994).

[22] J.P. Simonin, J. Phys. Chem. 99, 1577 (1995).

[23] H. Löwen, J. Phys.: Condens. Matter 10, L479 (1998).

[24] G. Téllez and T. Biben, Eur. Phys. J. E 2, 137 (2000).

[25] A.P. Philipse and G.H. Koenderink, Adv. Colloid Interface Sci. 100, 613 (2003). 